\title{
Senate baulks at cost of collider project
}

[WASHINGTON] The US Senate is moving to abandon the Next Linear Collider (NLC), a 30-kilometre long accelerator that highenergy physicists would like to build in the United States as their next major experiment after Europe's Large Hadron Collider.

The move follows a finding by the Department of Energy that the project could cost as much as $\$ 7.9$ billion to complete.

The Senate energy and water appropriations subcommittee, which monitors the department's budget, has called for NLC research costs to be cut from $\$ 14$ million to $\$ 6$ million next year. Senate staff say that the intention is to end funding altogether, but over two years, to minimize disruption at the Stanford Linear Accelerator Center (SLAC) in California, where most of the research is based.

The Senate move is motivated by a belief that the machine cannot be built in the foreseeable future, and that further research into its design is therefore pointless. One Senate staffer says that, even with large contributions from abroad, the NLC would cost the United States $\$ 5$ billion. "I cannot envisage any scenario in which the US will make that contribution," says the staffer.

Last month, after receiving a favourable review of the project together with the cost estimate, the energy department decided it was not ready to proceed with the conceptual

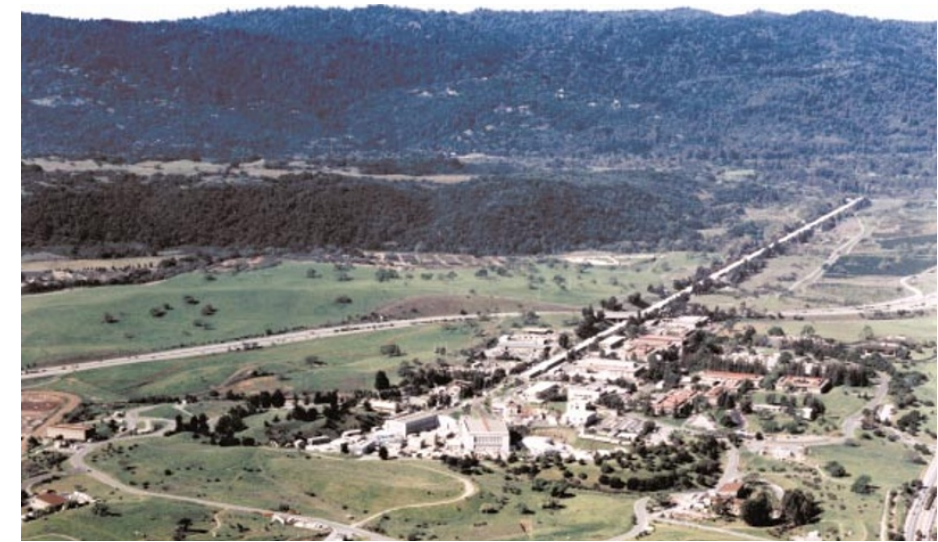

Cushioning the blow: Senators want to phase out linear collider research over two years to avoid too much disruption at the Stanford Linear Accelerator Center (left). design phase that is required before any decision is taken to build the collider.

According to Martha Krebs, assistant secretary of energy, it would "look premature" to start the conceptual design, which would cost about $\$ 70$ million over two years. "If you wait for a year or two you might actually have a chance to convince people that this is a good project," she says.

The energy and water appropriations subcommittee in the House of Representatives supports Krebs' plan to continue NLC research. A conference between representatives of the House and Senate subcommittees in September will determine the immediate fate of the project.

\section{Neurogenetic institute comes to California}

[SAN DIEGO] A Neurogenetic Institute with at least 30 faculty posts is to be set up at the University of Southern California (USC) with part of a $\$ 110$ million grant to the School of Medicine.

The grant, from the W. M. Keck Foundation, is one of the largest ever given to a US medical college. It is due to be announced today (29 July) along with plans to name the university's medical school after the foundation.

The Neurogenetic Institute will combine the efforts of $\mathbf{5 0}$ faculty members from within USC with the 30 to 35 new appointments. It will be housed in a research centre to be completed in two years on USC's Health Science Campus in East Los Angeles. USC officials estimate that onethird of the Keck grant will be used for the neurogenetic initiative.

Brian Henderson, a medical epidemiologist, will direct the institute, which will use an interdisciplinary approach to examine diseases such as Alzheimer's, Parkinson's, glaucoma, retinitis pigmentosa, macular degeneration, schizophrenia and manic depression.

"The challenge is to unravel the variables leading to neurological disorders and to obtain data on the malfunction of the expressed genes that cause disease," says Henderson, the former director of the USC/Kenneth Norris Jr Comprehensive Cancer Center.

Principal research goals for the institute will include sequencing and characterizing critical nervous system genes, characterizing genotypic variations, and determining their role in disorders of sensory, motor and cognitive functions. Other important goals include identifying the genetic mechanisms in the molecular pathogenesis of neuropsychiatric and neurological disorders to devise diagnostics and therapeutics, as well as linking genetic susceptibilities with environmental and psychological risk factors.

Henderson says the USC medical school has historically been oriented primarily towards clinical care, but is now seeking to enhance its research efforts. "What USC badly needs is research faculty," he says.

As a condition of the Keck grant, USC has agreed to raise an additional $\$ 330$ million for its medical school. Established in 1954 by the founder of Superior Oil, the Keck foundation of Los Angeles has previously given more than $\$ 140$ million to USC and its faculty members. Rex Dalton
But, even if collider research survives this year, the hesitancy of both the Congress and the administration to take the project further shows how difficult it will be for US physicists to build any machine with a price tag of much more than $\$ 1$ billion. The Senate move came despite the fact that Michael Witherell, the new director of Fermilab, outside Chicago, has buried the laboratory's rivalry with SLAC and sought to get involved in the NLC project.

The failure of even a unified high-energy physics community to generate momentum for the project confirms its waning influence, according to some observers in Washington. "There are a lot of other great ideas in science that don't cost billions of dollars," says one administration official.

"The American public doesn't care about staying at the forefront of high-energy physics." The official suggests that the end of the Cold War, together with declining public faith in the ability of physicists to come up with a clear explanation of the fundamental nature of matter, may help to explain reluctance to back the project.

Burt Richter, the director of SLAC, dismisses such arguments. He says that Congress initially approved the Superconducting Super Collider (SSC), at an estimated cost of $\$ 6$ billion, after the Cold War had ended.

Richter says the cost estimate for the NLC is highly conservative, to ensure that it will not drift up later. "The arguments now are not very different from the ones over the SSC," he says. "Every place in the world agrees that the next machine needs to be a big linear collider."

Witherell says: "What's needed is for the research to continue, so that we can work on things that will bring the cost down."

The NLC would accelerate electrons to $500 \mathrm{GeV}$, with the possibility of an upgrade that would take them to $1 \mathrm{TeV}$. The Stanford Linear Collider, the most powerful built so far, took electrons to $100 \mathrm{GeV}$. Scientists at KEK in Japan and at DESY in Germany are also researching colliders comparable to the NLC. Physicists hope for a global collaboration to build one machine, with the host bearing two-thirds of the cost. Colin Macilwain 